\title{
Weak-value amplification beyond the standard quantum limit in position measurements
}

\author{
Atsushi Nishizawa* \\ Theoretical Astrophysics 350-17, California Institute of Technology, Pasadena, California 91125, USA
}

(Received 30 April 2015; published 21 September 2015)

\begin{abstract}
In a weak measurement with postselection, a measurement value, called the weak value, can be amplified beyond the eigenvalues of an observable. However, there are some controversies whether weak-value amplification is practically useful in increasing the sensitivity of the measurement, in which fundamental quantum noise dominates. In this paper, we investigate the sensitivity limit of an optical interferometer when weak-value amplification is implemented, properly accounting for quantum shot noise and radiation-pressure noise. To do so, we formulate weak-value amplification in the Heisenberg picture instead of in the Schrödinger picture, which is conventionally used. This formulation enables us to understand intuitively what happens when the measurement outcome is postselected and the weak value is amplified. As a result, we find that the sensitivity limit is given by the standard quantum limit that is the same as in a standard interferometry. We also discuss a way to circumvent the standard quantum limit.
\end{abstract}

DOI: 10.1103/PhysRevA.92.032123

PACS number(s): 03.65.Ta, 42.50.Lc

\section{INTRODUCTION}

The idea of weak-value amplification (WVA) was originally introduced by Aharonov, Albert, and Vaidman (AAV) in 1988 [1] (see Ref. [2] for a review). When a system is weakly measured by a measuring device, the measurement results can be much larger than the eigenvalues of the observable by appropriately selecting initial and final states of the system. This theoretical prediction has been demonstrated in various pioneering experiments, e.g., the rotation of photon polarization $[3,4]$, the quantum-box problem [5], the arrival time of a single photon [6], the spin Hall effect of light [7], optical beam deflection [8,9], and optical phase [10]. For further recent theoretical and experimental developments regarding WVA, see the review papers [11-13].

The interesting nature of WVA results from the definition of the weak value:

$$
A_{w} \equiv \frac{\left\langle\psi_{f}|A| \psi_{i}\right\rangle}{\left\langle\psi_{f} \mid \psi_{i}\right\rangle},
$$

where $A$ is an observable associated with the system to be measured, and $\left|\psi_{i}\right\rangle$ and $\left|\psi_{f}\right\rangle$ are the initial and final states of the system. The weak value is interpreted as the observable evaluated at intermediate times between the preand postselections. From this definition, we see that if the pre- and postselected states are nearly orthogonal, it seems that the weak value becomes arbitrarily large. In fact, the experiments mentioned above have shown that WVA significantly improves the signal-to-noise ratio (SNR) in the situation where technical noise (e.g., alignment noise) dominates and outperforms standard interferometry. The theoretical study also confirms this advantage of WVA, see, e.g., Refs. [14,15], although it has been pointed out that the applicability of the amplification strongly depends on the property of the technical noise [16]. On the other hand, when the weak value becomes large, we must take into account the nonlinear effects of the von Neumann measurement [17-20]. As the result,

\footnotetext{
*anishi@caltech.edu
}

the amplification factor has a maximum value and vanishes when the pre- and postselected states are exactly orthogonal. Even in the weak-measurement regime, the nearly orthogonal postselection severely reduces output statistics, which consequently compensates the improvement of sensitivity due to the amplification of the signal. On the other hand, some authors $[21,22]$ have claimed that the sensitivity is infinitely improved by optimizing the wave functions of a system and a probe. However, if quantum shot noise in the detection process is taken into consideration, their conclusion would change. Therefore, the practical usefulness of WVA in increasing sensitivity has been often controversial.

In an optical interferometry, it was pointed out in Refs. [9,23] that, if photon shot noise dominates, there is no advantage of using WVA for improving the fundamental limit of parameter-estimation precision. From an informational approach with the quantum Fisher matrix [24-26], the same conclusion has also been obtained that WVA is suboptimal in enhancing the precision of parameter estimation and does not perform better than the standard statistical techniques (for the controversy on this conclusion, see also Refs. [27-29]). Indeed, these conclusions have been demonstrated in an optical experiment [30]. However, in an optical interferometry, not only shot noise but also radiation pressure noise contribute to fundamental quantum noise and leads to a kind of sensitivity limit, the so-called standard quantum limit (SQL) [31,32]. It is not clear how radiation-pressure noise affects the sensitivity limit of the measurement and alters the SQL from that in the standard interferometry when WVA is implemented. These questions are worth investigating because there are many systems in which the radiation pressure and the SQL play an important role, such as optomechanical systems [33,34] and gravitational-wave detection [35,36].

Conventionally, a weak measurement with postselection and WVA is formulated in the Schrödinger picture. However, this is somewhat less intuitive compared with the Heisenberg formulation and often leads to confusion. Also, it is more difficult to deal with moving mirrors and the radiation pressure noise in the Schrödinger picture. Instead, here we formulate WVA in the Heisenberg picture and deal with the quantum 
noise in a fully quantum-mechanical way, including the radiation-pressure noise. Then we derive the SQL and discuss a method to circumvent it.

This paper is organized as follows: In Sec. II, we briefly review position measurements in an optical interferometer and WVA in the Schrödinger picture. In Sec. III, we formulate WVA in the Heisenberg picture, appropriately taking into account quantum shot noise and radiation-pressure noise. The SQL is derived in Sec. IV and the method to overcome the SQL is presented in Sec. V. Finally, Sec. VI is devoted to a summary. In this paper, we use the unit of the speed of light $c=1$.

\section{WEAK-VALUE AMPLIFICATION OF A SIGNAL IN THE SCHRÖDINGER PICTURE}

We briefly review the Schrödinger formulation of WVA, which is the original formulation by Aharonov et al. [1] and which is conventionally used.

In this paper, we concentrate on a measurement of a small mirror displacement in an optical interferometer, particularly in a Michelson interferometer as shown in Fig. 1. This does not loose generality because a Michelson interferometer is geometrically equivalent to a Mach-Zehnder interferometer and a Sagnac interferometer and gives the same sensitivity limit for a position measurement.

We start with a single-photon case for an illustrative purpose. (We will generalize the result to a macroscopic beam later.) A photon is injected from the left side in Fig. 1, which is called the bright port because it is an input port of a laser light pulse in our setup. After being divided by the beam splitter, the photon takes two paths on the arms with unperturbed distance $L$. One of the photons in the $y$ arm is phase-shifted by $\theta$ (preselection), reflected at the mirror and sensing small displacements $\ell$ of the mirror (weak interaction), and recombined at the beam splitter with another photon that sensed small mirror displacement $-\ell$ in the $x$ arm (postselection). Finally, the photon is detected by the photodetector at the output (dark) port.

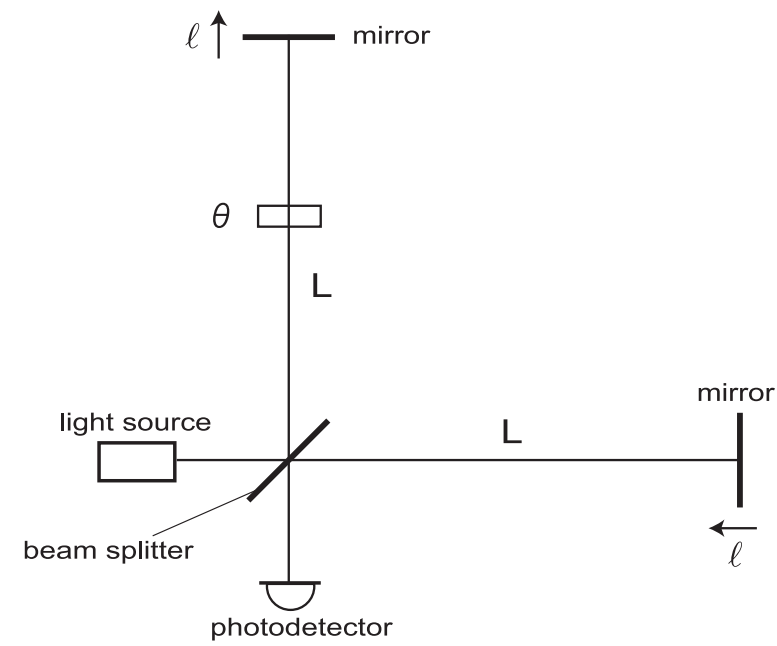

FIG. 1. Setup of a Michelson interferometer. Both arms have equal length $L$. The mirrors are displaced by small distance $\ell$ from $L$ differentially. The $\theta$ phase shifter is located in one of the arms.
In this optical setup, we are interested in the small differential displacement of the mirrors because a common mode can always be absorbed into the unperturbed arm length $L$. Then the observable can be expressed as $2 \ell A$, where the operator is $A \equiv|y\rangle\langle y|-| x\rangle\langle x|$ and the states $|y\rangle$ and $|x\rangle$ are those in which a photon propagates in the $y$ arm and $x$ arm, respectively. The operator $A$ has the eigenvalues \pm 1 , depending on the photon path, and carries which-path information of the photon. Therefore, we regard the beam's which-path information as the system to be measured via a weak measurement with coupling strength $2 \ell$. The initial preselected state of the system is denoted by

$$
\left|\psi_{i}\right\rangle=\frac{1}{\sqrt{2}}\left(e^{i \theta / 2}|y\rangle+e^{-i \theta / 2}|x\rangle\right)
$$

The initial phase offset $\theta$ is symmetrized merely for simplicity of calculation. An observable of an measuring device, or a pointer variable, is photon's momentum (frequency), which measures the phase shift induced by the mirror displacements in the Michelson interferometer. The initial state of the pointer is

$$
|\Phi\rangle=\int d p \Phi(p)|p\rangle .
$$

The interaction Hamiltonian is assumed to be instantaneous at time $t_{0}$ because the interaction time with a mirror is short enough compared with the dynamical timescale of the mirror:

$$
H=g \delta\left(t-t_{0}\right) A \otimes p .
$$

Here we defined $g \equiv-2 \ell$. This interaction Hamiltonian is viewed as a generalization of the von Neumann interaction like $\ell \otimes p$ to the Michelson-type interferometer. After the interaction given in Eq. (2) and the postselection by the final state of the system, $\left|\psi_{f}\right\rangle=(|y\rangle-|x\rangle) / \sqrt{2}$, the final state of the device can be exactly evaluated including nonlinear terms in the coupling [23],

$$
\begin{aligned}
\left|\Phi^{\prime}\right\rangle & =\left\langle\psi_{f}\left|e^{-i g A p}\right| \psi_{i}\right\rangle|\Phi\rangle \\
& =\int d p \Phi(p)|p\rangle\left\langle\psi_{f} \mid \psi_{i}\right\rangle\left(\cos g p-i A_{w} \sin g p\right) .
\end{aligned}
$$

The expectation value of the $n$th power of $p$ for the final state of the photon in Eq. (3) is given by [23]

$\left\langle p^{n}\right\rangle^{\prime}=\frac{\left\langle p^{n}\right\rangle+\left(\left|A_{w}\right|^{2}-1\right)\left\langle p^{n} \sin ^{2} g p\right\rangle+\operatorname{Im} A_{w}\left\langle p^{n} \sin 2 g p\right\rangle}{1+\left(\left|A_{w}\right|^{2}-1\right)\left\langle\sin ^{2} g p\right\rangle+\operatorname{Im} A_{w}\langle\sin 2 g p\rangle}$.

The brackets $\langle\cdots\rangle$ and $\langle\cdots\rangle^{\prime}$ denote averaging over the initial and final states of the measuring device, respectively. In our case, the weak value defined in Eq. (1) is

$$
A_{w}=-i \cot \frac{\theta}{2}
$$

and becomes significantly larger for small $\theta$. Then it seems that we have an amplified measurement value $2 \ell A_{w}$.

To explicitly see how the signal is amplified, we assume the initial momentum distribution of a photon to be non-zero-mean Gaussian (for multiple photons, a pulsed laser whose central 
frequency is mode-locked to $\left.\omega_{0}\right)$,

$$
\Phi(\omega)=\left(\frac{1}{2 \pi \sigma_{\omega}^{2}}\right)^{1 / 4} \exp \left[-\frac{\left(\omega-\omega_{0}\right)^{2}}{4 \sigma_{\omega}^{2}}\right] .
$$

Here we denoted momentum $p$ by angular frequency $\omega$ and will use this notation throughout this paper since we focus on an optical experiment. Substituting Eqs. (5) and (6) for Eq. (4), we obtain the following expressions for the first and second powers of $\omega-\omega_{0}:^{1}$

$$
\begin{gathered}
g\left\langle\omega-\omega_{0}\right\rangle^{\prime}=\frac{s e^{-s} \sin (\theta+\phi)}{1-e^{-s} \cos (\theta+\phi)}, \\
g^{2}\left\langle\left(\omega-\omega_{0}\right)^{2}\right\rangle^{\prime}=\frac{s}{2}\left[1+\frac{2 s e^{-s} \cos (\theta+\phi)}{1-e^{-s} \cos (\theta+\phi)}\right],
\end{gathered}
$$

where

$$
s \equiv 2 g^{2} \sigma_{\omega}^{2}=8 \sigma_{\omega}^{2} \ell^{2}, \quad \phi \equiv-2 g \omega_{0}=4 \omega_{0} \ell .
$$

The parameter $s$ characterizes the measurement strength, since large $g$ means strong coupling and large $\sigma_{\omega}$ means a narrow distribution of photons in the time domain. A weak measurement is defined by the opposite limit, $s \rightarrow 0$, which corresponds to two physical situations: (i) the measurement coupling, in this case mirror displacement, is small, (ii) the probe's wave function is narrow in momentum (frequency) space or broad in the spatial (time) domain.

At the limit of weak measurement, if only terms linear in $s$ are kept, the Eqs. (7) and (8) are reduced to

$$
\begin{aligned}
g\left\langle\omega-\omega_{0}\right\rangle^{\prime} & \approx s \cot (\theta+\phi), \\
g^{2}\left\langle\left(\omega-\omega_{0}\right)^{2}\right\rangle^{\prime} & \approx \frac{s}{2} .
\end{aligned}
$$

From these expressions, one finds that the frequency shift is proportional to $\cot (\theta+\phi)$ and could be largely amplified for small $\phi$ by taking small $\theta$. However, this result is for a single photon. In a sensitive position measurement in an optical experiment, a bunch of photons or an optical beam is usually used since the SNR is improved proportional to $\sqrt{N}$ for $N$ output photons. In this case, one needs to consider not only signal but also photon shot noise.

The above results can easily be extended to multiple photons. From Eq. (3), the probability distribution for a single photon at the output is

$$
\rho(\omega) \equiv \frac{\left|\left\langle\omega \mid \Phi^{\prime}\right\rangle\right|^{2}}{\left\langle\Phi^{\prime} \mid \Phi^{\prime}\right\rangle}
$$

For $N$ output photons, the photon-number distribution is simply given by ${ }^{2}$

$$
\bar{n}(\omega)=N \rho(\omega)
$$

\footnotetext{
${ }^{1}$ The sign of $\phi$ is different from that in Ref. [23]. This is just the matter of a different sign for mirror displacement.

${ }^{2}$ The extension of a wave function from one photon to multiple photons is somewhat conceptually strange in the Schrödinger picture. On the other hand, in the Heisenberg picture, as we discuss in Sec. III, the photon-number distribution at the output is more naturally introduced.
}

If we measure the shift of the pointer variable $\langle p\rangle^{\prime}$, its variance is given by $\operatorname{Var}[p]^{\prime}=\left\langle p^{2}\right\rangle^{\prime}-\left(\langle p\rangle^{\prime}\right)^{2}$ and is called frequency noise in Ref. [23] because it mainly comes from the initial momentum (frequency) distribution of a photon. On the other hand, the photon shot noise arises from fluctuations in photon number in each frequency and is shown in Ref. [23] to be given by $\left\langle p^{2}\right\rangle^{\prime}$. Therefore, the shot noise is always larger than the frequency noise and it is essential to consider the shot noise when we discuss sensitivity limit in an optical interferometer.

It was also found in Ref. [23] that there is a trade-off relation between the amplification factor of a weak value and the magnitude of the shot noise and that the SNR is not improved by WVA. However, in the discussion, they do not take into account radiation pressure noise, which is another fundamental quantum noise in an optical experiment and is essential when the SQL is discussed. To fully take into account the radiation pressure noise, we need to move to the Heisenberg picture.

\section{WEAK-VALUE AMPLIFICATION OF A SIGNAL IN THE HEISENBERG PICTURE}

When we deal with a radiation-pressure force, it is convenient to work in the Heisenberg picture. To do so, we use the single-photon formalism for a quantum electromagnetic field in an interferometer, in contrast to the two-photon formalism in Kimble et al. [35], because a pulsed laser has a broad spectrum around a central frequency. In the Heisenberg picture, an electric field is written as

$$
\begin{gathered}
E(t)=E^{(+)}(t)+E^{(-)}(t) \\
E^{(+)}(t)=\int_{0}^{\infty} \sqrt{\frac{2 \pi \hbar \omega}{\mathcal{A} c}} a_{\omega} e^{-i \omega t} \frac{d \omega}{2 \pi} \\
E^{(-)}=\left[E^{(+)}\right]^{\dagger}
\end{gathered}
$$

Here $\mathcal{A}$ is the effective scattering cross section of the beam, $a_{\omega}$ is the annihilation operator of a positive-frequency mode $\omega$ that satisfies the following commutation relations:

$$
\left[a_{\omega}, a_{\omega^{\prime}}\right]=0, \quad\left[a_{\omega}, a_{\omega^{\prime}}^{\dagger}\right]=2 \pi \delta\left(\omega-\omega^{\prime}\right) .
$$

Let us denote the input (incoming) and output (outgoing) fields at the dark port by $a$ and $b$, respectively. Suppose that a laser pulse is injected from the bright port and its field amplitude is denoted by $D=\alpha+d$ with a classical part $\alpha$ and a quantum fluctuating part $d$. For brevity, we omit the subscript $\omega$ for the field hereafter. The input-output relation for the fields is derived in Appendix A and is given up to linear order in vacuum fluctuations by

$b \approx i e^{2 i \omega L}\left\{\alpha \sin \omega \xi+\alpha \omega \xi_{\mathrm{r}} \cos \omega \xi+d \sin \omega \xi-i a \cos \omega \xi\right\}$,

with $\xi \equiv 2 \ell+\tau_{\theta}$ and

$$
\xi_{\mathrm{r}}=\frac{2 T}{m} \int_{0}^{\infty} \frac{d \omega}{2 \pi} \hbar \omega\left(\alpha a^{\dagger}+\alpha^{*} a\right) .
$$

Here, $\xi$ is a static time shift that pulsed light gains and $\tau_{\theta}$ is defined by $\theta / 2 \equiv \omega \tau_{\theta}(\omega)$ just for convenience of notation. The dynamic time shift $\xi_{\mathrm{r}}$ arises from the free motion of a mirror after the radiation pressure of the pulsed light is exerted on 
the mirror. In the expression, $m$ is the mirror mass and $T$ is the interval of measurements. The $T$ dependence shows up merely because the mirror moves freely after the disturbance of the previous measurement. In this derivation, we assumed $\left|\omega \xi_{\mathrm{r}}\right| \ll 1$ and neglected the higher-order terms in the vacuum fields and $\omega \xi_{\mathrm{r}}$.

The photon number at the output is

$$
\begin{aligned}
n= & b^{\dagger} b \\
\approx & |\alpha|^{2} \sin ^{2} \omega \xi+2|\alpha|^{2} \omega \xi_{\mathrm{r}} \cos \omega \xi \sin \omega \xi \\
& +\left(\alpha^{*} d+\alpha d^{\dagger}\right) \sin ^{2} \omega \xi+i\left(\alpha a^{\dagger}-\alpha^{*} a\right) \sin \omega \xi \cos \omega \xi
\end{aligned}
$$

Evaluating this in a coherent vacuum state $|0\rangle \equiv|0\rangle_{d}|0\rangle_{a}$, we have the average number of photons at the output

$$
\begin{aligned}
\bar{n}(\omega) & =\langle 0|n| 0\rangle \\
& =\bar{n}_{0}(\omega) \sin ^{2} \omega \xi,
\end{aligned}
$$

where we defined the average photon-number distribution at the input by $\bar{n}_{0}(\omega) \equiv|\alpha(\omega)|^{2}$.

In what follows, we consider the same Gaussian wave packet as in Eq. (6). However, it is not a wave function of a photon but here the photon-number distribution with amplitude $A$ in the Heisenberg picture:

$$
\alpha(\omega)=A \exp \left[-\frac{\left(\omega-\omega_{0}\right)^{2}}{4 \sigma_{\omega}^{2}}\right],
$$

for the frequency $\omega>0$. The width of the distribution should be $\sigma_{\omega} \lesssim \omega_{0}$. At $\omega \approx 0$, the distribution has an abrupt cutoff. This is somewhat artificial, but in most cases the cutoff is at the tail of the Gaussian distribution and does not much affect the observable signature at the output only if $\sigma_{\omega} \lesssim \omega_{0}$. Defining a sideband $\Omega$ by $\omega=\omega_{0}+\Omega$, and the normalized quantities, $\tilde{\Omega} \equiv \Omega / \omega_{0}$ and $\tilde{\sigma}_{\omega} \equiv \sigma_{\omega} / \omega_{0}$, the distribution is written as

$$
\alpha(\tilde{\Omega})=A \exp \left[-\frac{\tilde{\Omega}^{2}}{4 \tilde{\sigma}_{\omega}^{2}}\right],
$$

for $\tilde{\Omega} \geqslant-1$ and $\tilde{\sigma}_{\omega} \leqslant 1$. Then the average input power of a pulse is

$$
\begin{aligned}
P_{0} & =\int_{0}^{\infty} \frac{d \omega}{2 \pi} \hbar \omega \bar{n}_{0}(\omega) \\
& \approx \hbar \omega_{0}^{2}|A|^{2} \int_{-\infty}^{\infty} \frac{d \tilde{\Omega}}{2 \pi}(1+\tilde{\Omega}) e^{-\tilde{\Omega}^{2} / 2 \tilde{\sigma}_{\omega}^{2}} \\
& =\frac{1}{\sqrt{2 \pi}} \hbar \omega_{0} \sigma_{\omega}|A|^{2}
\end{aligned}
$$

At the second line, although the range of $\tilde{\Omega}$ is limited to $\tilde{\Omega} \geqslant$ -1 for the probe light, we extended the integral range to $-\infty \leqslant$ $\tilde{\Omega} \leqslant \infty$ because the contribution from the frequencies $\tilde{\Omega}<$ -1 is exponentially suppressed owing to the Gaussian tail.

By using the definition of $\phi$ in Eq. (9) and writing the phase factor in the signal as

$$
\omega \xi=(1+\tilde{\Omega}) \frac{\phi}{2}+\frac{\theta}{2},
$$

we have from Eq. (18) the averaged photon-number distribution at the output in the form

$$
\bar{n}(\tilde{\Omega})=\frac{\sqrt{2 \pi}}{\omega_{0} \tilde{\sigma}_{\omega}} N_{0} e^{-\tilde{\Omega}^{2} /\left(2 \tilde{\sigma}_{\omega}^{2}\right)} \sin ^{2}\left[\frac{\theta}{2}+(1+\tilde{\Omega}) \frac{\phi}{2}\right],
$$

where $N_{0} \equiv P_{0} /\left(\hbar \omega_{0}\right)$ is the effective total number of photons at the input. Note that the actual number of photons is different from $N_{0}$ because the light is not monochromatic. The total photon number at the output is obtained by integrating the photon distribution over all frequencies, $-1 \leqslant \tilde{\Omega} \leqslant \infty$. As well as the above integral in Eq. (21), we extend the integral range to $-\infty \leqslant \tilde{\Omega} \leqslant \infty$ and use the mathematical formulas for the integrals, Eqs. (B1) and (B2), we have the total number of photons at the output

$$
\begin{aligned}
N & \approx \omega_{0} \int_{-\infty}^{\infty} \frac{d \tilde{\Omega}}{2 \pi} \bar{n}(\tilde{\Omega}) \\
& =\frac{N_{0}}{2}\left[1-e^{-\tilde{\sigma}_{\omega}^{2} \phi^{2} / 2} \cos (\theta+\phi)\right] .
\end{aligned}
$$

The quantity $\tilde{\sigma}_{\omega}^{2} \phi^{2} / 2$ appearing in Eq. (24) coincides with the measurement-strength parameter $s$ in Eq. (9). When the measurement is weak $(s \ll 1)$ and the postselection is nearly orthogonal $(|\theta| \ll 1)$, the output photon number is significantly suppressed from the input photon number. On the other hand, for $|\theta| \ll 1$, the weak value defined in Eq. (5) is largely amplified. Therefore, we observe that there is a trade-off between the magnitude of the signal and its statistics.

Once we have the photon-number distribution at the output, it is straightforward to compute the frequency shift and its variance. The frequency shift is

$$
\begin{aligned}
\langle\tilde{\Omega}\rangle & \approx \omega_{0} \int_{-\infty}^{\infty} \frac{d \tilde{\Omega}}{2 \pi} \tilde{\Omega} \frac{\bar{n}(\tilde{\Omega})}{N} \\
& =\frac{2 s}{\phi} \frac{e^{-s} \sin (\theta+\phi)}{1-e^{-s} \cos (\theta+\phi)} .
\end{aligned}
$$

In the first line, we again approximately extended the integral range to $\tilde{\Omega} \geqslant-\infty$. Since $\langle\tilde{\Omega}\rangle \phi / 2=g\langle\Omega\rangle$, Eq. (25) coincides with the result in the Schrödinger picture in Eq. (7). In addition, the expectation value of the squared frequency shift is

$$
\begin{aligned}
\left\langle\tilde{\Omega}^{2}\right\rangle & \approx \omega_{0} \int_{-\infty}^{\infty} \frac{d \tilde{\Omega}}{2 \pi} \tilde{\Omega}^{2} \frac{\bar{n}(\tilde{\Omega})}{N} \\
& =\frac{2 s}{\phi^{2}}\left[1+\frac{2 s e^{-s} \cos (\theta+\phi)}{1-e^{-s} \cos (\theta+\phi)}\right] .
\end{aligned}
$$

Again this coincides with Eq. (8) obtained in the Schrödinger picture.

Figure 2 shows the photon-number distribution at the output $\bar{n}(\tilde{\Omega})$ in units of $N_{0} / \omega_{0}$, varying $\phi$ and $\theta$, respectively. As $\phi$ increases, the distribution is amplified and more shifted. On the other hand, as $\theta$ decreases or the weak value is more amplified, the distribution is more shifted but significantly suppressed. This behavior indicates the existence of the tradeoff relationship between the amplification of the weak value and the amount of output statistics. In Fig. 3, the photonnumber distribution is normalized and then is interpreted as a probability distribution. If one sees the probability 

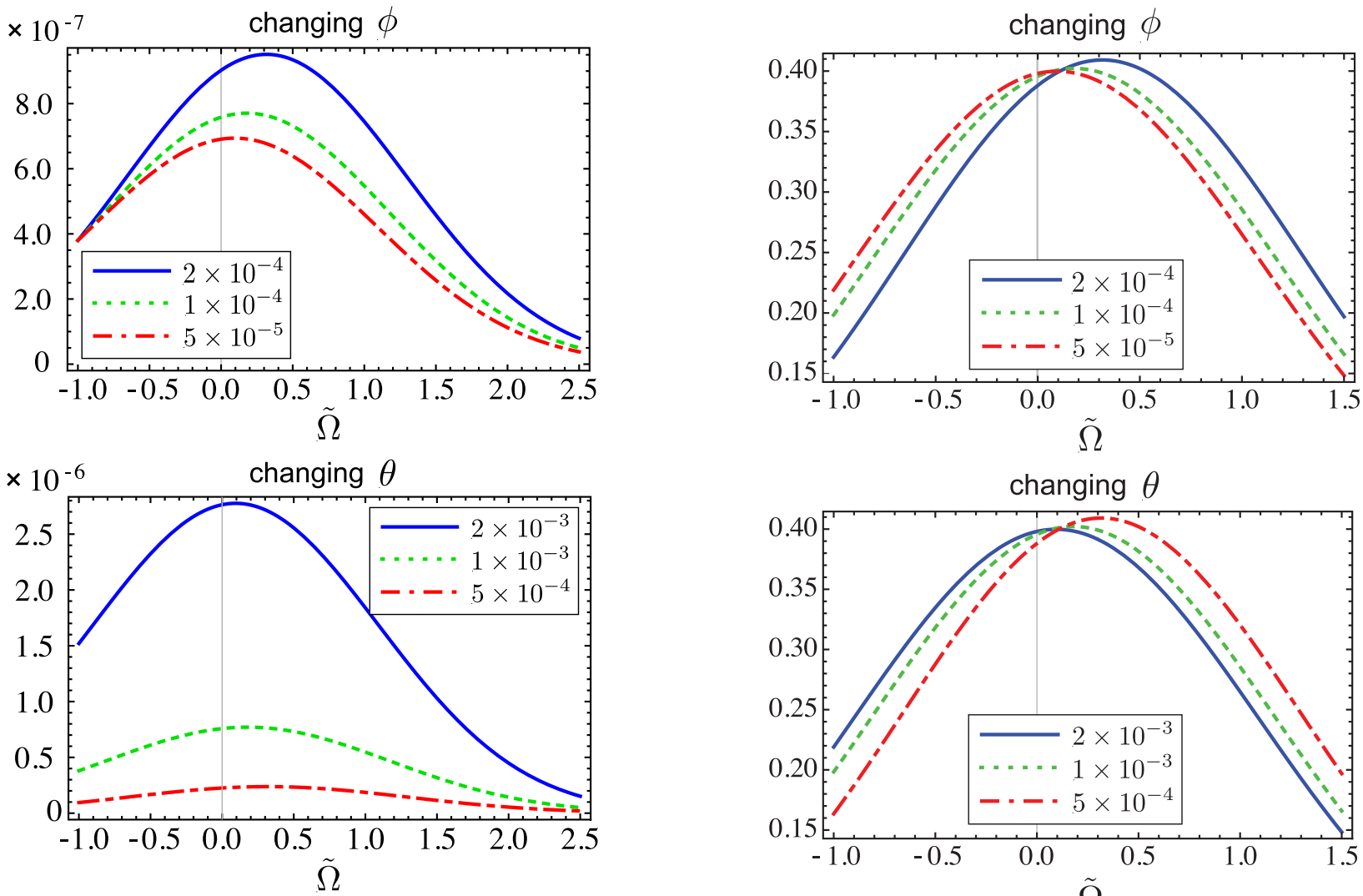

FIG. 2. (Color online) Photon-number distribution at the output $\bar{n}(\tilde{\Omega})$ in units of $N_{0} / \omega_{0}$. In each panel, one parameter is varied while fixing the fiducial parameters to $\tilde{\sigma}_{\omega}=1, \phi=10^{-4} \mathrm{rad}$, and $\theta=$ $10^{-3} \mathrm{rad}$. In the upper panel, $\phi=2 \times 10^{-4}$ (blue solid curve), $10^{-4}$ (green dotted curve), and $5 \times 10^{-5} \mathrm{rad}$ (red dotted-dashed curve). In the lower panel, $\theta=2 \times 10^{-3}$ (blue solid curve), $10^{-3}$ (green dotted curve), and $5 \times 10^{-4} \mathrm{rad}$ (red dotted-dashed curve).

distributions, the peak frequency is shifted without suppression of the amplitude. This feature often causes the confusion that SNR is amplified in the Schrödinger picture. However, this is not true. As Fig. 2 shows, the role of the postselection in the Heisenberg formulation is much clearer than that in the Schrödinger formulation.

In this section, we consider the average photon-number distribution at the output and calculate the frequency shift and its variance, based on the definitions in Eqs. (25) and (26). However, there should exist vacuum fluctuations of an electromagnetic field, which produce fluctuations in the photon number and consequently quantum noise, i.e., shot noise and radiation-pressure noise. To clarify the usefulness of WVA, we need to appropriately take into account quantum noise and evaluate the SNR. These will be done in the next section.

\section{QUANTUM NOISE AND STANDARD QUANTUM LIMIT}

In this section, we first introduce the quantum fluctuations of the photon number and compute shot noise and radiationpressure noise in a unified framework in the Heisenberg picture. Then, upon defining the SNR, we derive the SQL and the sensitivity limit for a position measurement.

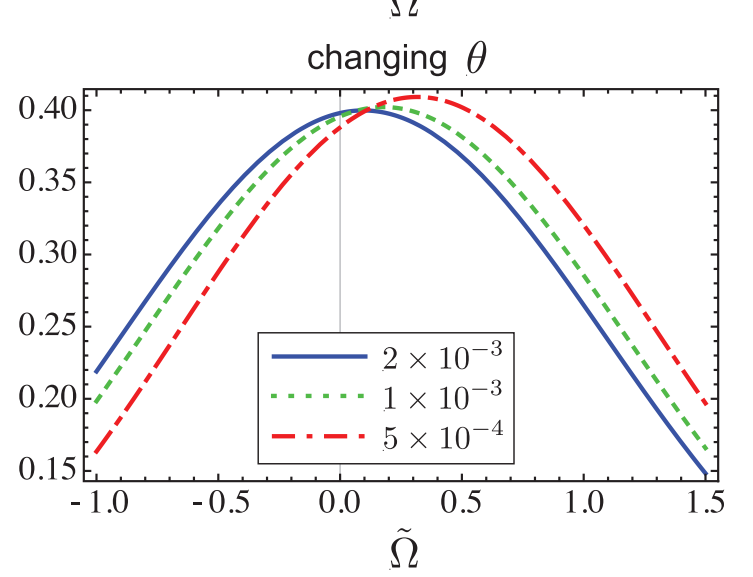

FIG. 3. (Color online) Probability distribution at the output $\omega_{0} \bar{n}(\tilde{\Omega}) /(2 \pi N)$. The parameters for each curve in each panel are the same as in Fig. 2, but they are normalized by the total photon number at the output.

From Eqs. (17) and (18), the fluctuating part of the photon number is

$$
\begin{aligned}
\Delta n(\omega) \equiv & n(\omega)-\bar{n}(\omega) \\
= & \left(\alpha^{*} d+\alpha d^{\dagger}\right) \sin ^{2} \omega \xi \\
& +\left\{2|\alpha|^{2} \omega \xi_{\mathrm{r}}+i\left(\alpha a^{\dagger}-\alpha^{*} a\right)\right\} \sin \omega \xi \cos \omega \xi
\end{aligned}
$$

Then the correction to the frequency shift due to deviation from the average photon number is given by

$$
\Delta \tilde{\Omega} \approx \omega_{0} \int_{-\infty}^{\infty} \frac{d \tilde{\Omega}}{2 \pi} \tilde{\Omega} \frac{\Delta n(\tilde{\Omega})}{N} .
$$

Note that $\Delta n$ and $\Delta \tilde{\Omega}$ above are operators. The expectation value of $\Delta \tilde{\Omega}$ is zero by definition. The quantum noise arises from the variance of $\Delta \tilde{\Omega}$, that is, $\left\langle(\Delta \tilde{\Omega})^{2}\right\rangle$.

Next, we evaluate $\left\langle(\Delta \tilde{\Omega})^{2}\right\rangle$. In our case, since each frequency mode of a laser pulse is independent when evaluated in a vacuum state $|0\rangle \equiv|0\rangle_{d}|0\rangle_{a}$, we have

$$
\left\langle\left\{\alpha d^{\dagger}+\alpha^{*} d\right\}\left\{\alpha^{\prime}\left(d^{\dagger}\right)^{\prime}+\left(\alpha^{*}\right)^{\prime} d^{\prime}\right\}\right\rangle=2 \pi \bar{n}_{0}(\omega) \delta\left(\omega-\omega^{\prime}\right),
$$

$\left\langle\left(\alpha a^{\dagger}-\alpha^{*} a\right)\left(\alpha^{\prime}\left(a^{\dagger}\right)^{\prime}-\left(\alpha^{*}\right)^{\prime} a^{\prime}\right)\right\rangle=-2 \pi \bar{n}_{0}(\omega) \delta\left(\omega-\omega^{\prime}\right)$. 
One needs to be careful that, in Eq. (27), the vacuum field $a$ and the vacuum field in $\xi_{\mathrm{r}}$ are defined at different times, because $\xi_{\mathrm{r}}$ is induced by a laser pulse of the previous measurement. To distinguish them, we use the subscript " 1 " for the vacuum field at the time of the previous laser pulse. By using Eq. (16), the expectation value of $\xi_{\mathrm{r}}^{2}$ is

$$
\begin{aligned}
\left\langle\xi_{\mathrm{r}}^{2}\right\rangle= & \left(\frac{2 T}{m}\right)^{2}\left\langle\int_{0}^{\infty} \frac{d \omega}{2 \pi} \hbar \omega\left(\alpha a_{1}^{\dagger}+\alpha^{*} a_{1}\right)\right. \\
& \left.\times \int_{0}^{\infty} \frac{d \omega^{\prime}}{2 \pi} \hbar \omega^{\prime}\left(\alpha^{\prime}\left(a_{1}^{\prime}\right)^{\dagger}+\left(\alpha^{\prime}\right)^{*} a_{1}^{\prime}\right)\right\rangle \\
= & \left(\frac{2 T}{m}\right)^{2} \int_{0}^{\infty} \frac{d \omega}{2 \pi}(\hbar \omega)^{2} \bar{n}_{0}(\omega) \\
\approx & \left(\frac{2 \hbar \omega_{0} T}{m}\right)^{2}\left(1+\tilde{\sigma}_{\omega}^{2}\right) N_{0} .
\end{aligned}
$$

Here we extended the integral range from $-\omega_{0}$ to $-\infty$ owing to the exponential suppression and used Eq. (21). As we prove below, there is no correlation between vacuum fields at different times. The correlation term between the vacuum fields in the same frequency mode but at different times by $T$ is

$$
\begin{aligned}
\left\langle\left\{\alpha a_{1}^{\dagger}+\alpha^{*} a_{1}\right\}\left\{\alpha a^{\dagger}-\alpha^{*} a\right\}+\left\{\alpha a^{\dagger}-\alpha^{*} a\right\}\left\{\alpha a_{1}^{\dagger}+\alpha^{*} a_{1}\right\}\right\rangle \\
=\left\langle\left\{\alpha a^{\dagger} e^{-i \omega T}+\alpha^{*} a e^{i \omega T}\right\}\left\{\alpha a^{\dagger}-\alpha^{*} a\right\}\right. \\
\left.\quad+\left\{\alpha a^{\dagger}-\alpha^{*} a\right\}\left\{\alpha a^{\dagger} e^{-i \omega T}+\alpha^{*} a e^{i \omega T}\right\}\right\rangle \\
=2 i|\alpha|^{2}\left\langle a a^{\dagger}\right\rangle \sin \omega T .
\end{aligned}
$$

Actually there exists a nonzero correlation proportional to $\sin \omega T$. However, in practice, the frequency resolution of a photodetector (multichannel CCD) is finite and a single frequency bin contains a number of frequency modes. Then summing over the modes averages out the phase of the correlation term. This contrasts conspicuously with a continuous measurement, in which the light spectrum is nearly monochromatic and the correlation term oscillates almost coherently with the phase difference of the order of $\tilde{\Omega} T \ll 1$. Thus, we conclude that we can treat the fields $a$ and $a_{1}$ independently in the pulsed measurements.

Therefore, from Eq. (27) and the above relations, we obtain

$$
\begin{aligned}
\left\langle\Delta n(\Omega) \Delta n\left(\Omega^{\prime}\right)\right\rangle= & 2 \pi \bar{n}_{0}(\omega) \sin ^{2} \omega \xi \delta\left(\omega-\omega^{\prime}\right) \\
& +\left\langle\xi_{r}^{2}\right\rangle \bar{n}_{0}(\omega) \bar{n}_{0}\left(\omega^{\prime}\right) \omega \omega^{\prime} \sin 2 \omega \xi \sin 2 \omega^{\prime} \xi \\
= & 2 \pi \frac{\bar{n}(\tilde{\Omega})}{\omega_{0}} \delta\left(\tilde{\Omega}-\tilde{\Omega}^{\prime}\right)+\omega_{0}^{2}\left\langle\xi_{r}^{2}\right\rangle \beta(\tilde{\Omega}) \beta\left(\tilde{\Omega}^{\prime}\right),
\end{aligned}
$$

with

$$
\beta(\tilde{\Omega}) \equiv \bar{n}_{0}(\tilde{\Omega})(1+\tilde{\Omega}) \sin [(1+\tilde{\Omega}) \phi+\theta] .
$$

The first term is shot noise, which results from the Poissonian statistics of photons and recovers Eq. (7) in Ref. [23]. The second term is radiation-pressure noise that we originally derived here in the context of WVA in the Heisenberg picture. Then the variance of the frequency shift is

$$
\begin{aligned}
\left\langle(\Delta \tilde{\Omega})^{2}\right\rangle & =\frac{\omega_{0}^{2}}{N^{2}} \int_{-\infty}^{\infty} \frac{d \tilde{\Omega}}{2 \pi} \int_{-\infty}^{\infty} \frac{d \tilde{\Omega}^{\prime}}{2 \pi} \tilde{\Omega} \tilde{\Omega}^{\prime}\left\langle\Delta n(\tilde{\Omega}) \Delta n\left(\tilde{\Omega}^{\prime}\right)\right\rangle \\
& \approx \frac{\left\langle\tilde{\Omega}^{2}\right\rangle}{N}+\frac{P_{0}^{2} \tilde{\sigma}_{\omega}^{4}}{N^{2} \hbar^{2}}\left\langle\xi_{\mathrm{r}}^{2}\right\rangle \sin ^{2} \theta \\
& \approx \frac{\left\langle(\Delta \tilde{\Omega})^{2}\right\rangle_{\mathrm{SQL}}}{2}\left[\frac{1}{I}+I\right] .
\end{aligned}
$$

In the second line, we assumed a weak measurement $(|\phi| \ll 1)$ and kept the leading-order terms in powers of small $\phi$. In the third line, we used Eq. (30) and the approximated photon number from Eq. (24),

$$
N \approx N_{0} \sin ^{2} \frac{\theta}{2}
$$

and defined

$$
\begin{gathered}
I \equiv N_{0} \eta \\
\eta \equiv \frac{4 \hbar \omega_{0} \sigma_{\omega} T}{m}\left|\cos \frac{\theta}{2}\right| \sqrt{1+\tilde{\sigma}_{\omega}^{2}}, \\
\left\langle(\Delta \tilde{\Omega})^{2}\right\rangle_{\mathrm{SQL}} \equiv 2 \tilde{\sigma}_{\omega}^{2} \eta\left|\sin \frac{\theta}{2}\right|^{-2} \\
=\frac{2 \tilde{\sigma}_{\omega}^{2} \eta}{|\cos (\theta / 2)|^{2}}\left|A_{w}\right|^{2} .
\end{gathered}
$$

The parameters $I$ and $\eta$ are interpreted as the intensity of a measurement and the susceptibility of a mirror to measurement disturbance, respectively. We see explicitly in the variance of Eq. (31) that there is a trade-off between shot noise and radiation-pressure noise, which consequently gives the lower bound for total noise. The minimum total noise is achievable when $I=1$, for which the noise is $\left\langle(\Delta \tilde{\Omega})^{2}\right\rangle_{\mathrm{SQL}}$. This sensitivity limit is the so-called SQL.

To derive minimum mirror displacement that is detectable by pulsed measurements, we define the SNR for a pointer shift:

$$
\mathrm{SNR} \equiv \frac{|\langle\tilde{\Omega}\rangle|}{\sqrt{\left\langle\Delta \tilde{\Omega}^{2}\right\rangle}} .
$$

Since the shift signal in Eq. (25) is expanded for small $\phi$ as

$$
|\langle\tilde{\Omega}\rangle| \approx \tilde{\sigma}_{\omega}^{2}\left|\phi \cot \frac{\theta}{2}\right|=\tilde{\sigma}_{\omega}^{2}\left|\phi A_{w}\right|,
$$

the optimal SNR is

$$
\mathrm{SNR}_{\mathrm{SQL}}^{2}=\frac{m}{8 T \hbar \omega_{0}^{2}}\left(\frac{\tilde{\sigma}_{\omega}^{2}}{1+\tilde{\sigma}_{\omega}^{2}}\right)^{1 / 2}\left|\cos \frac{\theta}{2}\right||\phi|^{2} .
$$

By using $|\phi|=4 \omega_{0} \ell$ and setting $\mathrm{SNR}=1$, we obtain the minimal detectable mirror displacement as

$$
\begin{aligned}
\ell_{\mathrm{SQL}} & =\left(1+\frac{1}{\tilde{\sigma}_{\omega}^{2}}\right)^{1 / 4} \sqrt{\frac{T \hbar}{2 m}}\left|\cos \frac{\theta}{2}\right|^{-1 / 2} \\
& \geqslant \sqrt{\frac{T \hbar}{2 m}} .
\end{aligned}
$$

This exactly coincides with the SQL derived from an elementary argument on two-time position measurements based on 
the Heisenberg uncertainty relation [37]. The sensitivity limit does not depend on the laser power of an experiment and gives a kind of fundamental limit imposed by the Heisenberg inequality when $T$ and $m$ are fixed. To increase the sensitivity further, one can choose a shorter time interval $T$ or a larger mass $m$. However, the SQL was achieved when $I=1$. To keep $I=1$ or keep $N_{0} T / m$ constant, one needs to increase $N_{0}$ to compensate the decrease in $T / m$. Namely, to improve tenfold the sensitivity to $\ell$, one needs to increase the laser power for a single pulse by $10^{2}$ times. This is the standard scaling appearing in quantum metrology when photons in each frequency are not correlated [38].

The interesting result here is that there is no amplification of the SNR due to WVA. Indeed, both the signal and noise are amplified via weak values. However, in the SNR formula, both amplifications are canceled out and the weak values disappear. This can be understood intuitively from the lower plot in Fig. 2. As $\theta$ decreases, the weak value is more amplified. However, the photon-number distribution is suppressed and becomes less sharp. Then the shot noise is amplified due to the small statistics at the output and the radiation pressure noise is also amplified by the same mechanism as the signal because these come from the displacements of a mirror. Therefore, not only signal but also quantum noise are amplified. If the distribution is normalized by $N$ as in Fig. 3, one sees only signal amplification, which often causes the confusion that WVA is useful for improving sensitivity.

We now comment on the experimental feasibility of the SQL in a pulsed measurement. The SQL is given for typical parameters by

$$
\ell_{\mathrm{SQL}} \geqslant 7.3 \times 10^{-18}\left(\frac{T}{10^{-3} \mathrm{~s}}\right)^{1 / 2}\left(\frac{1.0 \mathrm{~g}}{m}\right)^{1 / 2} \mathrm{~m} .
$$

To reach the SQL, we need $I=1$. For typical parameters, $I$ is written as

$$
\begin{aligned}
I \approx & 1.1 \times\left(\frac{1.0 \mathrm{~g}}{m}\right)\left(\frac{\omega_{0}}{1.8 \times 10^{15} \mathrm{~s}^{-1}}\right)\left(\frac{T}{10^{-3} \mathrm{~s}}\right)\left(\frac{P_{0}}{10 \mathrm{~J}}\right) \\
& \times\left(\frac{1+\tilde{\sigma}_{\omega}^{2}}{2}\right)^{1 / 2} \tilde{\sigma}_{\omega} \cos \frac{\theta}{2} .
\end{aligned}
$$

Since the requirement for these parameters is not so severe, the order of unity for $I$ and the SQL would be achievable with state-of-art technologies.

\section{QUANTUM TECHNIQUE TO OVERCOME THE STANDARD QUANTUM LIMIT}

There are many methods to overcome the SQL in a standard quantum metrology, e.g., by using quantum entanglement and a squeezed state [39]. These quantum correlations are also applicable to weak measurements with WVA and enhance the sensitivity of parameter estimation [40,41]. However, what the authors considered was not an optical interferometry with a bunch of photons but just multi-time measurements and is not relevant to our case in this paper. On the other hand, in some cases, such as the measurement of a coupling parameter between a spin- $\frac{1}{2}$ particle and coherent light, it is shown that the Heisenberg scaling can be achieved with classical coherent light $[42,43]$, although this does not necessarily mean that a weak measurement with a postselection outperforms standard interferometry.

In this section, we consider one example that circumvents the SQL in optical pulsed measurements; namely, the squeezed-vacuum input. This is implemented by replacing a coherent vacuum injected from the output port with a squeezed vacuum and evaluating the output photon distribution in the state

$$
\left|0_{s}\right\rangle=|0\rangle_{d} \otimes\left|0_{s}\right\rangle_{a},
$$

where $\left|0_{s}\right\rangle_{a}$ is the squeezed vacuum for the field $a$ and is defined by

$$
\left|0_{s}\right\rangle_{a} \equiv S\left(r_{s}, \phi_{s}\right)|0\rangle_{a},
$$

with the squeezing operator

$$
\begin{aligned}
S\left(r_{s}, \phi_{s}\right) \equiv & \exp \left[\int \frac{d \omega}{2 \pi} \frac{r_{s}(\omega)}{2}\right. \\
& \left.\times\left\{a^{2}(\omega) e^{-i \phi_{s}(\omega)}-\left[a^{\dagger}(\omega)\right]^{2} e^{i \phi_{s}(\omega)}\right\}\right] .
\end{aligned}
$$

Note that the squeezing factor $r_{s}$ and angle $\phi_{s}$ depend on frequency. However, for simplicity of notation, we omit the arguments of $r_{s}$ and $\phi_{s}$ in what follows. The annihilation and creation operators are converted by the squeezing operator as

$$
\begin{gathered}
S^{\dagger}\left(r_{s}, \phi_{s}\right) a S\left(r_{s}, \phi_{s}\right)=a \cosh r_{s}-a^{\dagger} e^{i \phi_{s}} \sinh r_{s}, \\
S^{\dagger}\left(r_{s}, \phi_{s}\right) a^{\dagger} S\left(r_{s}, \phi_{s}\right)=a^{\dagger} \cosh r_{s}-a e^{-i \phi_{s}} \sinh r_{s} .
\end{gathered}
$$

Since the squeezing operator does not change the linearity of $a$ and $a^{\dagger}$, we have

$$
{ }_{a}\left\langle 0_{s}|a| 0_{s}\right\rangle_{a}=0, \quad{ }_{a}\left\langle 0_{s}\left|a^{\dagger}\right| 0_{s}\right\rangle_{a}=0 .
$$

Then the quantities that are linear in $a$ and $a^{\dagger}$, e.g., $\bar{n}, N, \Delta n(\omega)$, $\langle\tilde{\Omega}\rangle$, and $\left\langle\tilde{\Omega}^{2}\right\rangle$, are the same as those in the coherent-vacuum case. However, the nonlinear terms in $a$ and $a^{\dagger}$, that is, shot noise and radiation-pressure noise, are modified.

The derivation of quantum noise is straightforward as in the previous section and is provided in Appendix C. The final expression when $|\phi| \ll 1$ is given by

$$
\begin{aligned}
\left\langle(\Delta \tilde{\Omega})^{2}\right\rangle_{s}= & \frac{\left\langle(\Delta \tilde{\Omega})^{2}\right\rangle_{\mathrm{SQL}}}{2} \\
& \times\left[\frac{1+F_{+}\left(r_{s 2}, \phi_{s 2}\right)}{I}+I\left\{1+f_{-}\left(r_{s 1}, \phi_{s 1}\right)\right\}\right],
\end{aligned}
$$

with

$$
\begin{aligned}
& f_{ \pm}\left(r_{s}, \phi_{s}\right)=2 \sinh r_{s}\left(\sinh r_{s} \pm \cos \phi_{s} \cosh r_{s}\right), \\
& F_{+}\left(r_{s}, \phi_{s}\right)=f_{+}\left(r_{s}, \phi_{s}\right) \cos ^{2} \frac{\theta}{2} .
\end{aligned}
$$

In the above equation, $I$ is the same as Eq. (33). We used the subscripts " 1 " and " 2 " to denote the first and the second laser pulses because $r_{s}$ and $\phi_{s}$ can take different values at different times. However, to obtain the analytic expression of shot noise and radiation-pressure noise, we assumed that the squeezing factor and squeezing angle for a single pulse are constant and independent of frequency. Of course, in practice, the squeezing 
factor and angle depend on frequency and are not reduced to the simple expression in Eq. (46). In that case, the quantum-noise spectrum is somewhat degraded and becomes closer to the conventional spectrum without the squeezing, depending on the amount of the imperfection and losses. Note that there is no correlation term between shot noise and radiation-pressure noise as in the case of a coherent vacuum because the vacuum fields at different times are not correlated.

Since the frequency shift in Eq. (36) is the same as in the coherent-vacuum case and does not depend on the squeezing parameters, it is convenient to define the ratio of noise in the squeezed-vacuum case to that in the coherent-vacuum case by

$$
R_{\mathrm{s}}^{2} \equiv \frac{\left\langle(\Delta \tilde{\Omega})^{2}\right\rangle_{s}}{\left\langle(\Delta \tilde{\Omega})^{2}\right\rangle_{\mathrm{SQL}}} .
$$

For specific choices of the squeezing angles,

$$
\begin{aligned}
& f_{ \pm}\left(r_{s}, 0\right)= \pm 2 \sinh r_{s} e^{ \pm r_{s}} \longrightarrow 1+f_{ \pm}=e^{ \pm 2 r_{s}}, \\
& f_{ \pm}\left(r_{s}, \pi\right)=\mp 2 \sinh r_{s} e^{\mp r_{s}} \longrightarrow 1+f_{ \pm}=e^{\mp 2 r_{s}} .
\end{aligned}
$$

If $\theta$ is small, $F_{+} \approx f_{+}$. Then for a set of squeezing parameters $r_{s}=r_{s 1}=r_{s 2}$ and $\phi_{s}=\phi_{s 1}=\phi_{s 2}=0$,

$$
R_{\mathrm{s}}^{2}=\frac{1}{2}\left[\frac{e^{+2 r_{s}}}{I}+I e^{-2 r_{s}}\right],
$$

and for $r_{s}=r_{s 1}=r_{s 2}$ and $\phi_{s}=\phi_{s 1}=\phi_{s 2}=\pi$,

$$
R_{\mathrm{s}}^{2}=\frac{1}{2}\left[\frac{e^{-2 r_{s}}}{I}+I e^{+2 r_{s}}\right] .
$$

In these cases, one of the shot noise or radiation-pressure noise is reduced and the other is enhanced. This means that an effective laser power changes to $I e^{-2 r_{s}}$ in the former case and $I e^{+2 r_{s}}$ in the latter case, and that the optimal laser power to reach the SQL also changes. However, the quantum noise never circumvents the SQL. If one chooses the squeezing angle optimally as $\phi_{s 1}=0$ and $\phi_{s 2}=\pi$, the noise is minimized:

$$
R_{\mathrm{s}}^{2}=\frac{e^{-2 r_{s}}}{2}\left[\frac{1}{I}+I\right] \text {. }
$$

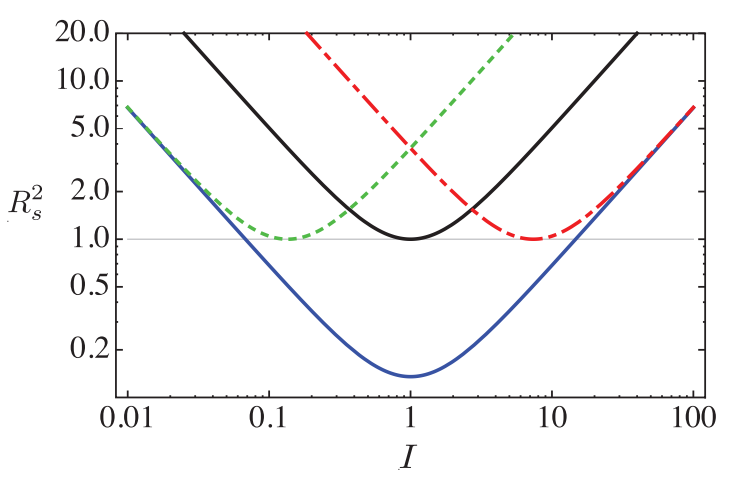

FIG. 4. (Color online) Ratio of quantum noise $R_{s}^{2}$ with and without the squeezed-vacuum input. The black solid curve is the conventional case $\left(r_{s}=0\right)$. The other curves are when the squeezing factor is set to $r_{s}=1$ and the squeezing angles are $\left(\phi_{s 1}, \phi_{s 2}\right)=(0,0)$ (green dotted curve), $(\pi, \pi)$ (red dotted-dashed curve), and $(0, \pi)$ (blue solid curve).
Therefore, we can overcome the SQL by a factor of $e^{-r_{s}}$ in the SNR. In Fig. 4, we show quantum noise as a function of $I$ for different choices of squeezing angle.

\section{CONCLUSIONS}

We have studied WVA in position measurements in an optical interferometer by appropriately accounting for quantum shot noise and radiation-pressure noise in the Heisenberg picture. The Heisenberg formulation enables us to intuitively understand what happens when measurement outcomes are postselected and the weak value is amplified. As discussed in Sec. III, the photon-number distribution as a function of frequency is shifted by the WVA but is significantly suppressed due to the postselection. However, once this distribution is normalized by the total number of photons, the suppression of the statistics disappears. Therefore, it looks like a signal is amplified without any cost. This often leads to a wrong conclusion. However, working in the Heisenberg picture makes such a misunderstanding clear, as we have explicitly shown in Figs. 2 and 3.

Then we have taken into account vacuum fluctuations of an electromagnetic field and derived quantum noises, i.e., shot noise and radiation pressure noise, in Eq. (31). By defining the SNR, we have shown that the measurement sensitivity of a mirror position is limited by the SQL in Eq. (37), which is the same formula as that in a standard interferometry. Interestingly, both the signal in Eq. (36) and the quantum noise in Eq. (35) are amplified by the WVA. As a result, there is no amplification in the SNR because both the amplifications are canceled out and the weak value disappears in the SNR formula. We have also shown that the SQL can be overcome by implementing the squeezed-vacuum input.

Therefore, we conclude that WVA has no advantage to improve the sensitivity of position measurements when quantum noise dominates. However, it is still possible to circumvent the SQL if other quantum techniques such as squeezing and entanglement are combined with WVA.

\section{ACKNOWLEDGMENTS}

The author is supported by JSPS Postdoctoral Fellowships for Research Abroad. He thanks M.-K. Fujimoto and K. Nakamura for fruitful discussions at the early stage of this work, and also thanks Yanbei Chen and Yiqiu Ma for carefully reading the manuscript and giving comments.

\section{APPENDIX A: INPUT-OUTPUT RELATION}

We derive an optical input-output relation for the Michelson interferometer shown in Fig. 5.

\section{Field relationship at beam splitter}

At the beam splitter, the output field $b$ is related with the other fields $c_{x}^{\prime}$ and $c_{y}^{\prime}$ by

$$
E_{b}(t)=\frac{1}{\sqrt{2}}\left[E_{c_{y}^{\prime}}(t)-E_{c_{x}^{\prime}}(t)\right] .
$$




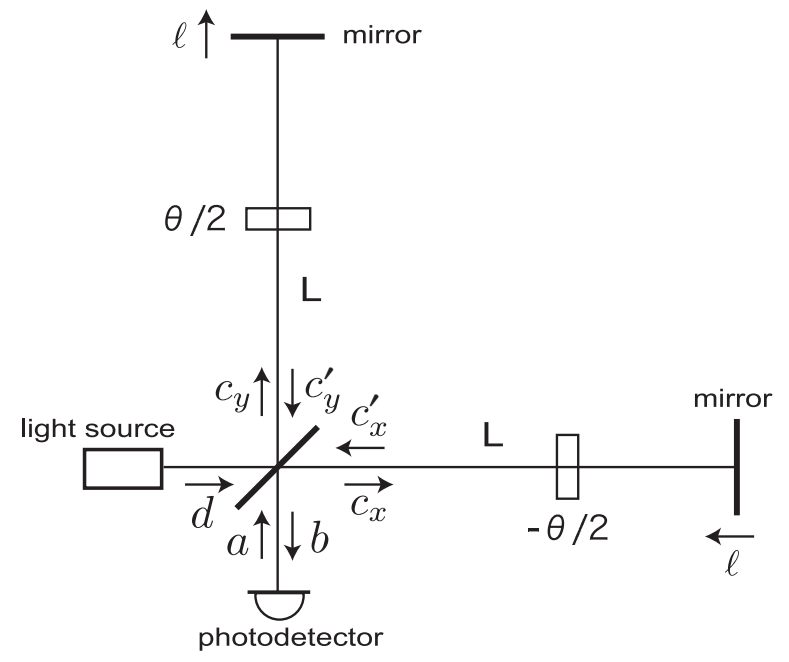

FIG. 5. Electric fields in a Michelson interferometer. Here the initial phase offset $\theta$ is symmetrized merely for simplicity of calculation.

Using Eqs. (12)-(14), we have the relationship

$$
b=\frac{1}{\sqrt{2}}\left(c_{y}^{\prime}-c_{x}^{\prime}\right)
$$

On the other hand, the fields $c_{x}, c_{y}$ are expressed in terms of the input fields $D$ and $a$ as

$$
c_{y}=\frac{1}{\sqrt{2}}(D+a), \quad c_{x}=\frac{1}{\sqrt{2}}(D-a) .
$$

\section{Field relationship in arms}

When light goes around an interferometer arm whose length is $L$, the light takes time of a round trip in each arm, $2 L$. Other than this, the light also undergoes the short time delay due to small displacement of a mirror, $2\left(\ell+\ell_{\mathrm{r}}\right)$, and a phase offset by a phase shifter (for a round trip), $\tau_{\theta}$. The mirror displacement $\ell$ is defined as a differential component of mirror displacement between both arms because a common mode can always be included in the definition of $L$. The difference in the definitions between $\ell$ and $\ell_{\mathrm{r}}$ is that the former is the mirror displacement without radiation pressure and the latter is due to radiation-pressure noise induced by vacuum field fluctuations. The $\tau_{\theta}$ is defined by $\theta / 2 \equiv \omega \tau_{\theta}(\omega)$ and the initial phase offset $\theta$ between two arms is symmetrized merely as in Fig. 5 for simplicity of a calculation. For the other arm, the signs of $\ell$, $\ell_{\mathrm{r}}$, and $\tau_{\theta}$ are reversed.

Denoting the time delays as

$$
\xi \equiv 2 \ell+\tau_{\theta}, \quad \xi_{\mathrm{r}} \equiv 2 \ell_{\mathrm{r}},
$$

the electromagnetic fields departing from the beam splitter and returning there after the reflections at the mirrors are connected by the relations

$$
\begin{aligned}
& E_{c_{y}^{\prime}}(t)=E_{c_{y}}\left[t-2 L-\left(\xi+\xi_{\mathrm{r}}\right)\right], \\
& E_{c_{x}^{\prime}}(t)=E_{c_{x}}\left[t-2 L+\left(\xi+\xi_{\mathrm{r}}\right)\right] .
\end{aligned}
$$

Note that $\xi_{\mathrm{r}}$ is a Hermitian operator. Then using Eqs. (12)-(14), we have the relationship

$$
c^{\prime}=c e^{i \omega\left(2 L \pm \xi \pm \xi_{\mathrm{r}}\right)},
$$

where the upper and lower signs correspond to the $y$ and $x$ arms, respectively.

\section{Radiation-pressure noise}

The number distributions of photons impinging on the mirror in each arm of an interferometer are given by

$$
\begin{aligned}
& n_{y} \equiv c_{y}^{\dagger} c_{y}=\frac{1}{2}\left(D^{\dagger} D+a^{\dagger} D+D^{\dagger} a\right), \\
& n_{x} \equiv c_{x}^{\dagger} c_{x}=\frac{1}{2}\left(D^{\dagger} D-a^{\dagger} D-D^{\dagger} a\right),
\end{aligned}
$$

where we ignored the higher-order terms in vacuum fluctuations and keep up to the first-order terms in the amplitude of vacuum fluctuations. We will do as well hereafter. Defining $D=\alpha+d$ by separating a classical part $\alpha$ and a quantum fluctuating part $d$, we have the average photon number $\bar{n}_{x}=$ $\bar{n}_{y}=|\alpha|^{2} / 2$ and their fluctuations

$$
\begin{aligned}
\Delta n_{y} & \equiv n_{y}-\bar{n}_{y} \\
& =\frac{1}{2}\left(\alpha d^{\dagger}+\alpha^{*} d+\alpha a^{\dagger}+\alpha^{*} a\right), \\
\Delta n_{x} & \equiv n_{x}-\bar{n}_{x} \\
& =\frac{1}{2}\left(\alpha d^{\dagger}+\alpha^{*} d-\alpha a^{\dagger}-\alpha^{*} a\right) .
\end{aligned}
$$

Since the classical part can be compensated exactly by feedback controls, we do not consider it in the following. The momentum fluctuation exerted on the mirror per a pulsed light (twice due to reflection) is

$$
\Delta P_{i}=2 \int_{0}^{\infty} \frac{d \omega}{2 \pi} \hbar \omega \Delta n_{i}(\omega), \quad i=x, y .
$$

Let a pulse interval be $T$. Then at the next measurement time after the interval $T$, neglecting the contribution from a common mode, the mirror changes its position by

$$
\begin{aligned}
\xi_{\mathrm{r}} & = \pm 2 \ell_{\mathrm{r}} \\
& =\frac{2 T \Delta P_{y, x}}{m} \\
& = \pm \frac{2 T}{m} \int_{0}^{\infty} \frac{d \omega}{2 \pi} \hbar \omega\left(\alpha a^{\dagger}+\alpha^{*} a\right) .
\end{aligned}
$$

The upper sign is for the $y$ arm and the lower sign is for the $x$ arm.

\section{Electromagnetic field at the output}

Combining Eqs. (A1)-(A3), the output field is

$$
b=e^{2 i \omega L}\left\{i D \sin \omega\left(\xi_{r}+\xi\right)+a \cos \omega\left(\xi_{r}+\xi\right)\right\} .
$$

Here we assumed $\left|\omega \xi_{r}\right| \ll 1$ and that a higher-order term $a \times \xi_{r}$ can be neglected. Thus, plugging in $D=\alpha+d$ and neglecting the higher-order terms, we obtain

$b \approx i e^{2 i \omega L}\left\{\alpha \sin \omega \xi+\alpha \omega \xi_{r} \cos \omega \xi+d \sin \omega \xi-i a \cos \omega \xi\right\}$. 


\section{APPENDIX B: INTEGRAL FORMULAS}

We use the following integral formulas:

$$
\begin{aligned}
\int_{-\infty}^{\infty} e^{-a x^{2}} \cos (2 b x+c) d x & =\sqrt{\frac{\pi}{a}} e^{-b^{2} / a} \cos c \\
\int_{-\infty}^{\infty} e^{-a x^{2}} \sin (2 b x+c) d x & =\sqrt{\frac{\pi}{a}} e^{-b^{2} / a} \sin c .
\end{aligned}
$$

\section{APPENDIX C: DERIVATION OF QUANTUM NOISE IN THE CASE OF SQUEEZED-VACUUM INPUT}

Since the squeezing operator in Eq. (42) does not change the linearity of $a$ and $a^{\dagger}$, we have

$$
{ }_{a}\left\langle 0_{s}|a| 0_{s}\right\rangle_{a}=0, \quad{ }_{a}\left\langle 0_{s}\left|a^{\dagger}\right| 0_{s}\right\rangle_{a}=0 .
$$

Then the quantities that are linear in $a$ and $a^{\dagger}$, e.g., $\bar{n}, N, \Delta n(\omega)$, $\langle\tilde{\Omega}\rangle$, and $\left\langle\tilde{\Omega}^{2}\right\rangle$, are the same as those in the coherent-vacuum case. However, the nonlinear terms in $a$ and $a^{\dagger}$ are modified:

$$
\begin{aligned}
{ }_{a}\left\langle 0_{s}\left|a^{\dagger}\left(a^{\prime}\right)^{\dagger}\right| 0_{s}\right\rangle_{a} & =-2 \pi \delta\left(\omega-\omega^{\prime}\right) e^{-i \phi_{s}} \sinh r_{s} \cosh r_{s}, \\
{ }_{a}\left\langle 0_{s}\left|a a^{\prime}\right| 0_{s}\right\rangle_{a} & =-2 \pi \delta\left(\omega-\omega^{\prime}\right) e^{i \phi_{s}} \sinh r_{s} \cosh r_{s}, \\
{ }_{a}\left\langle 0_{s}\left|a\left(a^{\prime}\right)^{\dagger}\right| 0_{s}\right\rangle_{a} & =2 \pi \delta\left(\omega-\omega^{\prime}\right) \cosh ^{2} r_{s}, \\
{ }_{a}\left\langle 0_{s}\left|a^{\dagger} a^{\prime}\right| 0_{s}\right\rangle_{a} & =2 \pi \delta\left(\omega-\omega^{\prime}\right) \sinh ^{2} r_{s} .
\end{aligned}
$$

The quadratic terms that arise from Eq. (27) and give shot noise and radiation-pressure noise have the expectation values

$$
\begin{aligned}
{ }_{a}\left\langle 0_{s}\right| & \left\{\alpha a^{\dagger}+\alpha^{*} a\right\}\left\{\alpha^{\prime}\left(a^{\prime}\right)^{\dagger}+\left(\alpha^{\prime}\right)^{*} a^{\prime}\right\}\left|0_{s}\right\rangle_{a} \\
= & 2 \pi \delta\left(\omega-\omega^{\prime}\right)\left\{\bar{n}_{0}(\omega) \cosh 2 r_{s}-\operatorname{Re}\left[\alpha^{2} e^{-i \phi_{s}}\right] \sinh 2 r_{s}\right\}, \\
& \times{ }_{a}\left\langle 0_{s}\left|\left\{\alpha a^{\dagger}-\alpha^{*} a\right\}\left\{\alpha^{\prime}\left(a^{\prime}\right)^{\dagger}-\left(\alpha^{\prime}\right)^{*} a^{\prime}\right\}\right| 0_{s}\right\rangle_{a} \\
= & -2 \pi \delta\left(\omega-\omega^{\prime}\right)\left\{\bar{n}_{0}(\omega) \cosh 2 r_{s}+\operatorname{Re}\left[\alpha^{2} e^{-i \phi_{s}}\right] \sinh 2 r_{s}\right\},
\end{aligned}
$$

and

$$
\begin{aligned}
\left\langle\xi_{r}^{2}\right\rangle_{s} \equiv & a_{1}\left\langle 0_{s}\left|\xi_{r}^{2}\right| 0_{s}\right\rangle_{a_{1}} \\
= & \left(\frac{2 T}{m}\right)^{2} \int_{0}^{\infty} \frac{d \omega}{2 \pi} \int_{0}^{\infty} \frac{d \omega^{\prime}}{2 \pi} \hbar \omega \hbar \omega^{\prime} \\
& \times{ }_{a_{1}}\left\langle 0_{s}\left|\left\{\alpha a_{1}^{\dagger}+\alpha^{*} a_{1}\right\}\left\{\alpha^{\prime}\left(a_{1}^{\prime}\right)^{\dagger}+\left(\alpha^{\prime}\right)^{*} a_{1}^{\prime}\right\}\right| 0_{s}\right\rangle_{a_{1}} \\
= & \left(\frac{2 T}{m}\right)^{2} \int_{0}^{\infty} \frac{d \omega}{2 \pi}(\hbar \omega)^{2} \\
& \times\left\{|\alpha|^{2} \cosh 2 r_{s 1}-\operatorname{Re}\left[\alpha^{2} e^{-i \phi_{s 1}}\right] \sinh 2 r_{s 1}\right\} .
\end{aligned}
$$

As in the case of a coherent vacuum, there is no correlation between vacuum fields at different times because of a broadband spectrum.

In the above, $\alpha^{2}$ always appears in the product $\alpha^{2} e^{-i \phi_{s}}$. Without loss of generality, we can assume that $\alpha$ is real by absorbing the phase into $\phi_{s}$. So we write

$$
\cos \phi_{s}=\frac{\operatorname{Re}\left[\alpha^{2} e^{-i \phi_{s}}\right]}{|\alpha|^{2}}, \quad \sin \phi_{s}=-\frac{\operatorname{Im}\left[\alpha^{2} e^{-i \phi_{s}}\right]}{|\alpha|^{2}} .
$$

In addition, we distinguish $r_{s}$ and $\phi_{s}$ at different times by using the subscripts " 1 " and " 2 " for the first and the second laser pulses, respectively. To obtain the analytic expression of shot noise and radiation-pressure noise, we assume that the squeezing factor and squeezing angle are constant and independent of frequency. Combining all these and using Eq. (27), we obtain

$$
\begin{aligned}
\left\langle\Delta n(\omega) \Delta n\left(\omega^{\prime}\right)\right\rangle_{s} & =2 \pi \bar{n}_{0}(\omega) \sin ^{2} \omega \xi \delta\left(\omega-\omega^{\prime}\right)\left\{1+f_{+}\left(r_{s 2}, \phi_{s 2}\right) \cos ^{2} \omega \xi\right\}+\left\langle\xi_{r}^{2}\right\rangle_{s} \bar{n}_{0}(\omega) \bar{n}_{0}\left(\omega^{\prime}\right) \omega \omega^{\prime} \sin 2 \omega \xi \sin 2 \omega^{\prime} \xi \\
& =2 \pi \frac{\bar{n}(\tilde{\Omega})}{\omega_{0}} \delta\left(\tilde{\Omega}-\tilde{\Omega}^{\prime}\right)\left\{1+f_{+}\left(r_{s 2}, \phi_{s 2}\right) \cos ^{2}\left[(1+\tilde{\Omega}) \frac{\phi}{2}+\frac{\theta}{2}\right]\right\}+\left\langle\xi_{r}^{2}\right\rangle_{s} \omega_{0}^{2} \beta(\tilde{\Omega}) \beta\left(\tilde{\Omega}^{\prime}\right),
\end{aligned}
$$

with

$$
\begin{aligned}
f_{ \pm}\left(r_{s}, \phi_{s}\right) & \equiv 2 \sinh r_{s}\left(\sinh r_{s} \pm \cos \phi_{s} \cosh r_{s}\right), \\
\left\langle\xi_{r}^{2}\right\rangle_{s} & =\left\langle\xi_{r}^{2}\right\rangle\left\{1+f_{-}\left(r_{s 1}, \phi_{s 1}\right)\right\} .
\end{aligned}
$$

Then an additional term to the shot noise is

$$
\begin{aligned}
& \frac{\omega_{0}^{2}}{N^{2}} \int_{-\infty}^{\infty} \frac{d \tilde{\Omega}}{2 \pi} \int_{-\infty}^{\infty} \frac{d \tilde{\Omega}^{\prime}}{2 \pi} \tilde{\Omega} \tilde{\Omega}^{\prime} 2 \pi \frac{\bar{n}(\tilde{\Omega})}{\omega_{0}} \delta\left(\tilde{\Omega}-\tilde{\Omega}^{\prime}\right) f_{+}\left(r_{s 2}, \phi_{s 2}\right) \cos ^{2}\left[(1+\tilde{\Omega}) \frac{\phi}{2}+\frac{\theta}{2}\right] \\
& =\frac{\sqrt{2 \pi} N_{0}}{4 \tilde{\sigma}_{\omega} N^{2}} f_{+}\left(r_{s 2}, \phi_{s 2}\right) \int_{-\infty}^{\infty} \frac{d \tilde{\Omega}}{2 \pi} \tilde{\Omega}^{2} e^{-\tilde{\Omega}^{2} / 2 \tilde{\sigma}_{\omega}^{2}} \sin ^{2}[(1+\tilde{\Omega}) \phi+\theta] \\
& =\frac{\left\langle\tilde{\Omega}^{2}\right\rangle}{N} F_{+}\left(r_{s 2}, \phi_{s 2}\right),
\end{aligned}
$$

where

$$
\begin{aligned}
F_{+}\left(r_{s 2}, \phi_{s 2}\right) & \equiv \frac{\tilde{\sigma}_{\omega}^{2}}{8 N\left\langle\tilde{\Omega}^{2}\right\rangle} N_{0} f_{+}\left(r_{s 2}, \phi_{s 2}\right)\left\{1-e^{-2 \phi^{2} \tilde{\sigma}_{\omega}^{2}}\left(1-4 \phi^{2} \tilde{\sigma}_{\omega}^{2}\right) \cos [2(\phi+\theta)]\right\} \\
& \approx f_{+}\left(r_{s 2}, \phi_{s 2}\right) \cos ^{2} \frac{\theta}{2}
\end{aligned}
$$


In the second line, we expanded in small $\phi$ and took the leading-order term. The radiation-pressure term is simply given by replacing $\left\langle\xi_{r}^{2}\right\rangle$ in the calculation of the coherent-vacuum case with

$$
\left\langle\xi_{r}^{2}\right\rangle_{s}=\left\{1+f_{-}\left(r_{s 1}, \phi_{s 1}\right)\right\}\left\langle\xi_{r}^{2}\right\rangle .
$$

Thus,

$$
\begin{aligned}
\left\langle(\Delta \tilde{\Omega})^{2}\right\rangle_{s} & =\frac{\omega_{0}^{2}}{N^{2}} \int_{-\infty}^{\infty} \frac{d \tilde{\Omega}}{2 \pi} \int_{-\infty}^{\infty} \frac{d \tilde{\Omega}^{\prime}}{2 \pi} \tilde{\Omega} \tilde{\Omega}^{\prime}\left\langle\Delta n(\tilde{\Omega}) \Delta n\left(\tilde{\Omega}^{\prime}\right)\right\rangle \\
& \approx \frac{\left\langle\tilde{\Omega}^{2}\right\rangle}{N}\left\{1+F_{+}\left(r_{s 2}, \phi_{s 2}\right)\right\}+\frac{N_{0}^{2} \omega_{0}^{2} \tilde{\sigma}_{\omega}^{4}}{N^{2}}\left\langle\xi_{\mathrm{r}}^{2}\right)\left\{1+f_{-}\left(r_{s 1}, \phi_{s 1}\right)\right\} \sin ^{2} \theta \\
& \approx \frac{\left\langle(\Delta \tilde{\Omega})^{2}\right\rangle_{\mathrm{SQL}}}{2}\left[\frac{1+F_{+}\left(r_{s 2}, \phi_{s 2}\right)}{I}+I\left\{1+f_{-}\left(r_{s 1}, \phi_{s 1}\right)\right\}\right] .
\end{aligned}
$$

In the third line, we used Eqs. (30) and (32), expanded in small $\phi$.

[1] Y. Aharonov, D. Z. Albert, and L. Vaidman, Phys. Rev. Lett. 60, 1351 (1988).

[2] Y. Aharonov and L. Vaidman, in Lecture Notes in Physics, edited by J. G. Muga, R. S. Mayato, and 1. L. Egusquiza (Springer Verlag, Berlin, 2007), Vol. 734, p. 399.

[3] N. W. M. Ritchie, J. G. Story, and R. G. Hulet, Phys. Rev. Lett. 66, 1107 (1991).

[4] G. J. Pryde, J. L. O’Brien, A. G. White, T. C. Ralph, and H. M. Wiseman, Phys. Rev. Lett. 94, 220405 (2005).

[5] K. J. Resch, J. S. Lundeen, and A. M. Steinberg, Phys. Lett. A 324, 125 (2004).

[6] Q. Wang, F.-W. Sun, Y.-S. Zhang, Jian-Li, Y.-F. Huang, and G.-C. Guo, Phys. Rev. A 73, 023814 (2006).

[7] O. Hosten and P. Kwiat, Science 319, 787 (2008).

[8] P. B. Dixon, D. J. Starling, A. N. Jordan, and J. C. Howell, Phys. Rev. Lett. 102, 173601 (2009).

[9] D. J. Starling, P. B. Dixon, A. N. Jordan, and J. C. Howell, Phys. Rev. A 80, 041803 (2009).

[10] D. J. Starling, P. B. Dixon, N. S. Williams, A. N. Jordan, and J. C. Howell, Phys. Rev. A 82, 011802 (2010).

[11] A. G. Kofman, S. Ashhab, and F. Nori, Phys. Rep. 520, 43 (2012).

[12] J. Dressel, M. Malik, F. M. Miatto, A. N. Jordan, and R. W. Boyd, Rev. Mod. Phys. 86, 307 (2014).

[13] Y. Shikano, arXiv:1110.5055.

[14] N. Brunner and C. Simon, Phys. Rev. Lett. 105, 010405 (2010).

[15] A. N. Jordan, J. Martínez-Rincón, and J. C. Howell, Phys. Rev. X 4, 011031 (2014).

[16] G. C. Knee and E. M. Gauger, Phys. Rev. X 4, 011032 (2014).

[17] A. Di Lorenzo and J. C. Egues, Phys. Rev. A 77, 042108 (2008).

[18] X. Zhu, Y. Zhang, S. Pang, C. Qiao, Q. Liu, and S. Wu, Phys. Rev. A 84, 052111 (2011).

[19] K. Nakamura, A. Nishizawa, and M.-K. Fujimoto, Phys. Rev. A 85, 012113 (2012).

[20] T. Koike and S. Tanaka, Phys. Rev. A 84, 062106 (2011).

[21] A. D. Parks and J. E. Gray, Phys. Rev. A 84, 012116 (2011).
[22] Y. Susa, Y. Shikano, and A. Hosoya, Phys. Rev. A 85, 052110 (2012).

[23] A. Nishizawa, K. Nakamura, and M.-K. Fujimoto, Phys. Rev. A 85, 062108 (2012).

[24] S. Tanaka and N. Yamamoto, Phys. Rev. A 88, 042116 (2013).

[25] C. Ferrie and J. Combes, Phys. Rev. Lett. 112, 040406 (2014).

[26] J. Combes, C. Ferrie, Z. Jiang, and C. M. Caves, Phys. Rev. A 89, 052117 (2014).

[27] L. Vaidman, arXiv:1402.0199.

[28] Y. Kedem, arXiv:1402.1352.

[29] C. Ferrie and J. Combes, arXiv:1402.2954.

[30] G. I. Viza, J. Martínez-Rincón, G. B. Alves, A. N. Jordan, and J. C. Howell, arXiv:1410.8461.

[31] V. B. Braginsky and Y. I. Vorontsov, Sov. Phys. Usp. 17, 644 (1975).

[32] C. Caves, K. Thorne, R. Drever, V. Sandberg, and M. Zimmermann, Rev. Mod. Phys. 52, 341 (1980).

[33] M. Aspelmeyer, T. J. Kippenberg, and F. Marquardt, Rev. Mod. Phys. 86, 1391 (2014).

[34] M. Poot and H. S. J. van der Zant, Phys. Rep. 511, 273 (2012).

[35] H. J. Kimble, Y. Levin, A. B. Matsko, K. S. Thorne, and S. P. Vyatchanin, Phys. Rev. D 65, 022002 (2001).

[36] S. L. Danilishin and F. Y. Khalili, Living Rev. Relativity 15, 5 (2012).

[37] V. B. Braginsky, F. Y. Khalili, and K. S. Thorne, Quantum Measurement (Cambridge University Press, Cambridge, UK, 1992).

[38] V. Giovannetti, S. Lloyd, and L. Maccone, Science 306, 1330 (2004).

[39] C. M. Caves, Phys. Rev. D 23, 1693 (1981).

[40] S. Pang, J. Dressel, and T. A. Brun, Phys. Rev. Lett. 113, 030401 (2014).

[41] S. Pang and T. A. Brun, arXiv:1409.2567.

[42] L. Zhang, A. Datta, and I. A. Walmsley, Phys. Rev. Lett. 114, 210801 (2015).

[43] A. N. Jordan, J. Tollaksen, J. E. Troupe, J. Dressel, and Y. Aharonov, Quantum Stud.: Math. Found. 2, 5 (2015). 